\title{
THE IMPACT OF POINT CLOUD DENSITY ON BUILDING OUTLINE EXTRACTION
}

\author{
U. Drešček ${ }^{1, *}$, M. Kosmatin Fras ${ }^{1}$, A. Lisec ${ }^{1}$, D. Grigillo ${ }^{1}$ \\ ${ }^{1}$ University of Ljubljana, Faculty of Civil and Geodetic Engineering, Jamova cesta 2, 1000 Ljubljana, Slovenia - (urska.drescek, \\ mojca.kosmatin-fras, anka.lisec, dejan.grigillo)@fgg.uni-lj.si
}

Commission II, WG II/4

KEY WORDS: UAV, point cloud, density, building outline extraction, quality analysis

\begin{abstract}
:
Recently, building outline extraction from point cloud has gained momentum in particular in the context of 3D building modelling based on a data-driven approach, which has also been our motivation. For an accurate building outline extraction from a point cloud, various factors affecting the quality should be considered. In this research, we analysed the influence of point cloud density on the quality of the extracted building outlines. The input data was a classified photogrammetric point cloud, obtained from the dense image matching of images acquired by an optical sensor mounted on the unmanned aerial vehicle (UAV). For outline extraction, we selected two procedures, namely the direct approach and the raster approach. In the direct approach, building outlines are extracted directly from the points that have been classified as buildings. First, a convex hull with the alpha algorithm is estimated, which is further generalised with the Douglas-Peucker algorithm. This is followed by the shape regularisation to ensure perpendicular angles of the outline. In the raster approach, we first rasterised the building points and then extracted the building outlines using the Hough transform. In both approaches, the result is a roof outline in a 2D plane representing the maximum extent of the building above the surface. The building outlines were extracted from point clouds with five different densities. For both approaches, the quality assessment has shown that point cloud density has an impact on the building outline extraction, especially on the completeness of the outlines.
\end{abstract}

\section{INTRODUCTION}

Georeferenced point clouds, generated from data acquired by optic or laser sensors mounted on aerial or terrestrial platforms, have become the essential source for geospatial data modelling. We have been witnessing the advances in the development of algorithms for automatic or semi-automatic extraction of spatial entities from georeferenced point clouds with particular attention to building outline extraction (Gilani et al., 2016; Haala and Kada, 2010; Kaartinen et al., 2005; Pfeifer et al., 2007; Rottensteiner et al., 2014). With the advances in 3D city modelling, this topic has gained particular attention again in the geospatial research and innovation domain. Namely, there are several potential applications where 3D city models with building models can be used: from urban planning, navigation, facility management, 3D cadastre to various environmental simulation (Biljecki et al., 2015).

For building outline extraction and 3D city modelling, a point cloud could be obtained either by airborne laser scanning (ALS) or by dense image matching using aerial images. An aerial acquired georeferenced point cloud provides information about the position, shape, and orientation of a building in the scene. Some authors suggested 3D building modelling approaches using ALS point cloud data to detect building outlines and later to reconstruct $3 \mathrm{D}$ building models (Dorninger and Pfeifer, 2008; Jarzabek-Rychard and Maas, 2017). In our research, we have focused on the photogrammetric point cloud, which is obtained by dense image matching of highly overlapping images, acquired by the optical sensor mounted on the unmanned aerial vehicle (UAV). Comparing to ALS, the UAV is a low-cost and more flexible source to obtain the point cloud. There have been some publications on using UAV data for building outline extraction (Dai et al., 2017), and for 3D building modelling ( $\mathrm{Li}$ et al., 2016; Malihi et al., 2018). However, there are still many challenges of using low-cost UAV-based geospatial data acquisition for building outline extractions as well as 3D modelling of the built environment.

In general, the process of building reconstruction from point cloud includes several steps, which are building detection, outline extraction, roof reconstruction, model generation and regularisation, and model quality analysis (Dorninger and Pfeifer, 2008). For an accurate 3D building model, an adequate building outline should be generated. Some reconstruction methods rely on outlines from additional sources, such as cadastre or topography database. However, many times auxiliary building outlines are not available, or they are not accurate enough. In the so-called data-driven 3D building modelling based on the point cloud, we need to extract the building outlines directly from the point cloud. Zhang et al. (2006) created coarse outlines connecting the LiDAR points that were denoted as a roof and shapes were simplified. The building outlines were also defined by merging neighbouring planes that were reconstructed from points representing the roof (Awrangjeb and Lu, 2014; Habib et al., 2010). Various algorithms have been further applied for building outline extraction from point clouds, e.g. random sample consensus (RANSAC) (Jarzabek-Rychard, 2012), Hough transform (Widyaningrum et al., 2019), convex hull (Sampath and Shan, 2007), alpha shape (dos Santos et al., 2019) or combinations of them (Albers et al., 2016). A comprehensive review of the

\footnotetext{
Corresponding author
} 
methods for building outline extraction from ALS point clouds has been provided by Tomljenović et al. (2015).

The objective of our research has been to analyse the influence of point cloud density on the quality of the building outline extraction. For this purpose, we decided on two different approaches to extract building outlines from a UAV photogrammetric point cloud and analysed the impact of various point cloud density on the accuracy of building outlines, which were extracted by the selected algorithms. The building outline has been defined as an external boundary of the roof, which represents the maximum $2 \mathrm{D}$ extent of the building above the Earth surface.

The paper is organised as follows. In Section 2, we present the methodology, i.e. describe the used dataset, two selected approaches for outline extraction utilised in this research, and methods for quality assessment. In Section 3, the results of building outline extraction are presented, with the quality assessment focusing on the impact of point cloud density on building outline extraction. In Section 4, we make conclusions with the main findings and suggestions for further research.

\section{METHODOLOGY}

The generalised processing workflow that has been used to analyse the impact of UAV photogrammetric point cloud density on the extracted building outlines is schematically presented in Figure 1, where operations are given in rectangles and data is denoted with grey shapes.

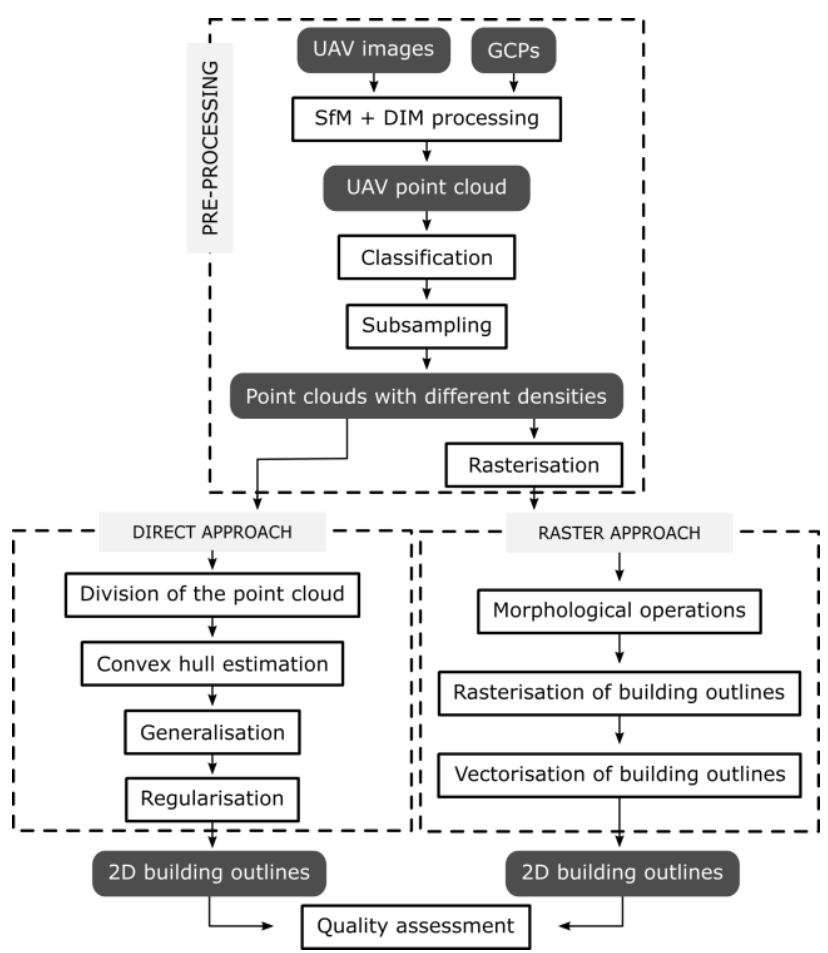

Figure 1. The processing workflow for building outline extraction based on UAV photogrammetric point cloud.

\subsection{Dataset Used}

We used the UAV photogrammetric point cloud as an input dataset for analysing the impact of point cloud density on the quality of the building outline extraction. To obtain the point cloud, we conducted a field survey with the optic camera mounted on a UAV platform. The UAV images were acquired in the rural area in the central part of Slovenia, near the village of Vače, with approximate area coverage of 220 x $220 \mathrm{~m}^{2}$ (Figure 2). The case study refers to the buildings in a small village, where family houses and agriculture auxiliary buildings are present. Although the village is small, it is an interesting study area for our research. Namely, there are various typical roof shapes, which are also very common in the Slovenian villages and small towns.

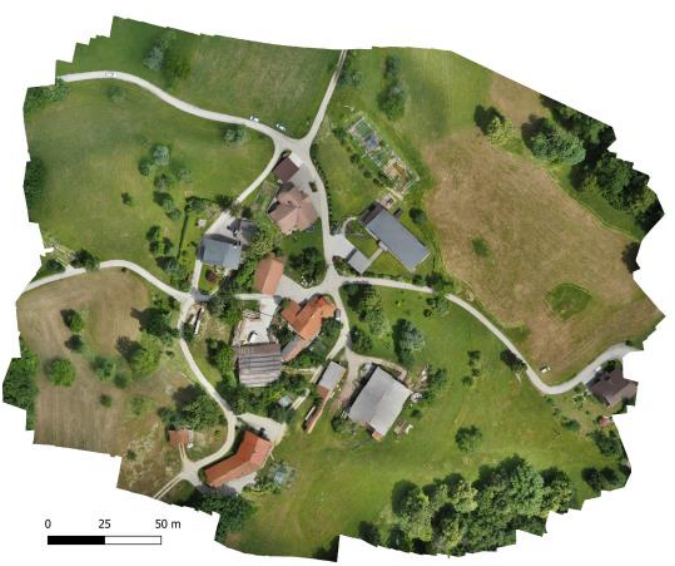

Figure 2. Orthomosaic of the study area.

The UAV flight took place on June 8, 2018, using Sky Hero X8 octocopter. The images were captured with the digital camera Olympus PEN E-PL7. The flight was conducted in 30 minutes around the noontime, acquiring 344 nadir images. Table 1 shows the detailed specifications of UAV data acquisition.

\begin{tabular}{|l|c|}
\hline Settings & \\
\hline Location & Vače, Slovenia \\
\hline Area & $\sim 5$ ha \\
\hline UAV model & Sky Hero X8 octocopter \\
\hline Focal length & $17 \mathrm{~mm}$ \\
\hline Camera resolution & $16.1 \mathrm{Mpx}$ \\
\hline Sensor size & $4608 \times 3456 \mathrm{px}$ \\
\hline Pixel size & $3.74 \mu \mathrm{m}$ \\
\hline Overlap (forward/sideward) & $85 / 65 \%$ \\
\hline Flight altitude & $50 \mathrm{~m}$ \\
\hline Ground sampling distance & $1 \mathrm{~cm} / \mathrm{px}$ \\
\hline Number of images & 344 \\
\hline
\end{tabular}

Table 1. Specification for UAV data acquisition.

The overlapping nadir images were indirectly georeferenced using ground control points (GCPs). The total number of 9 GCPs were evenly distributed on the perimeter and in the middle of the study area. All GCPs were signalized with artificial circular targets with a diameter of $20 \mathrm{~cm}$ and surveyed before UAV data acquisition. The GCPs were surveyed with RTK (Real-Time Kinematic) method, using GNSS (Global Navigation Satellite System) Leica Viva receiver. The positions of GCPs were determined in the Slovenian national reference system (D96/TM, SVS2010) - for this purpose, corrections of GNSS observations were considered by linking the survey with the Slovenian permanent GNSS stations network (SIGNAL).

\subsection{Data Pre-processing}

Data pre-processing was conducted in order to generate the UAV photogrammetric point cloud, to classify the point cloud 
in desired semantic classes, and to extract the points representing buildings. We also subsampled these point clouds aiming to produce different point clouds with smaller density and then rasterised them.

Firstly, the UAV images were processed in the photogrammetric software package Agisoft Photoscan Professional v. 1.4.3. The procedure utilises algorithms of Structure-from-Motion (SfM) and dense image matching (DIM), which allow obtaining the dense photogrammetric point cloud. Secondly, the dense photogrammetric point cloud was classified within the same software, using build-in algorithms for point cloud classification. For the point cloud that contained only points corresponding to the buildings, some misclassified points were manually reclassified. The manual work was needed to provide quality input data for further processing because we wanted to exclude the impact of point cloud classification on the results of building outline extraction.

The point cloud containing only points of buildings was subsampled to derive point clouds with different point densities. The subsampling was done in the open-source software Cloud Compare v2.11 using the tool Subsample. The density of the point cloud was defined according to the minimum distance between the points. For each point, the surrounding points that were within the predefined 3D neighbourhood were removed. We prepared the data for building outline extraction for 5 different point cloud densities, having the point cloud density equal to $1,2.5,5,10$, and $20 \mathrm{~cm}$. These point clouds were used as input data for building outline extraction with the direct approach described in Section 2.3.

The last step of the data pre-processing was rasterisation of all these 5 point clouds. The rasterized point clouds were the input for the raster outline extraction approach. The rasterisation was done in the spatial ETL environment, FME by SafeSoftware using build-in transformer ImageRasterizer. For each point cloud of different density, one raster was made with spatial resolution equal to the point cloud density. The result of rasterisation was a binary image, representing 1 for buildings and 0 for the background.

\subsection{Direct Approach}

The first approach for the outline extraction is referred to as a direct approach because it determines the outline directly from the point cloud. The whole procedure for the direct approach was developed in the spatial ETL (Extract, Transform, Load) environment, using the FME by SafeSoftware. The input data for this procedure is the classified UAV photogrammetric point cloud containing only points corresponding to the class Buildings.

The direct approach consists of four steps (Figure 3):

- Division of the point cloud: the initial point cloud is divided into individual groups of points, where each group represents one building.

- Concave hull estimation: for each group of points, a concave hull is created, which represents the $2 \mathrm{D}$ polygon as an approximate outline of the building. The alpha value for the creation of the hull has been empirically set to a value 0.4 .

- Generalisation: each concave hull is generalised with the Douglas-Peucker algorithm with a tolerance value of 0.2 . The algorithm removes the vertices of the outline polygon that are within the specified tolerance, the remaining vertices preserve its location.

- Regularisation: created 2D building outlines are regularised with a function Regularize Building Footprints. The function was developed for commercial software package ArcGIS Pro by ESRI, and it can be integrated into FME processing workflow. The building outlines are processed using the Right Angle method, which ensures perpendicular angles of the building outlines by moving the polygon vertices into the desired location within the specified tolerance. The method requires two parameters: the tolerance, which is defined by the maximum deviations of the regularised outline to the initial ones (in our case was set to 0.25 ), and the precision, which is defined by the size of the grid used for regularisation (in our case was set to the value of 0.15 ).

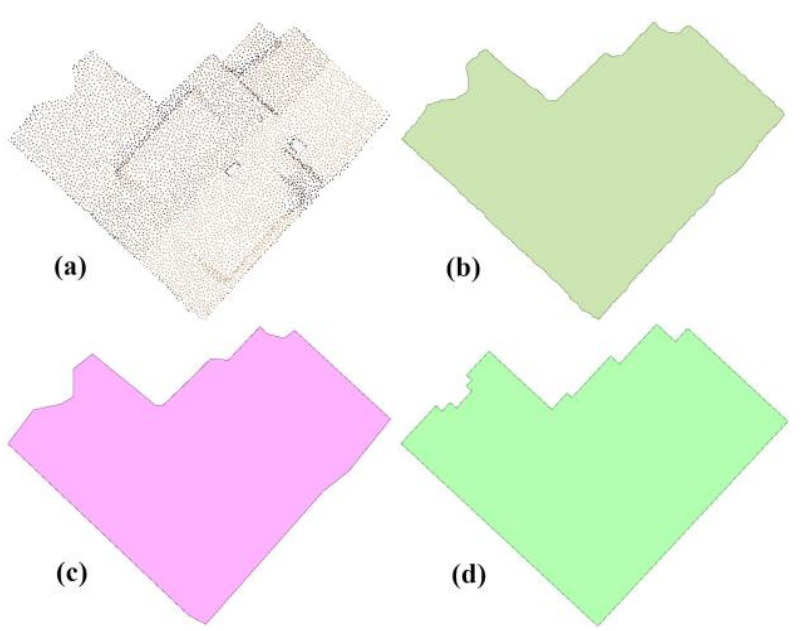

Figure 3. The results of building outline extraction obtained by the direct approach for the selected building: (a) a point cloud of the building; (b) a convex hull; (c) a generalised outline; (d) a regularised outline.

The values of the parameters in the direct approach were set empirically according to the data set used in our analysis.

\subsection{Raster Approach}

The procedure for the raster approach is developed in Matlab. Building outlines are vectorised from the rasterised point cloud using the following steps (Figure 4):

- Morphological operations: to obtain homogenous building areas, morphological close using structure element square with the dimension 2 (dimension 4 in case of $1 \mathrm{~cm}$ raster) has been applied to raster images and followed by closing of the remaining holes.

- Creation of rasterised building outlines: building outlines are obtained by tracing the exterior boundaries of the object in the image. For that purpose, we used Matlab function boundaries (Gonzalez et al., 2004).

- Vectorisation of building outlines: building outlines are vectorised using Hough transform separately for each object within the building outlines image. The minimal distance between detected parallel lines was set to $0.5 \mathrm{~m}$. The vectorisation is in detail discussed in Grigillo and Kanjir (2012). 


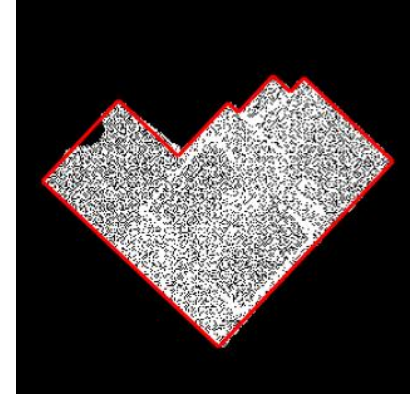

(a)

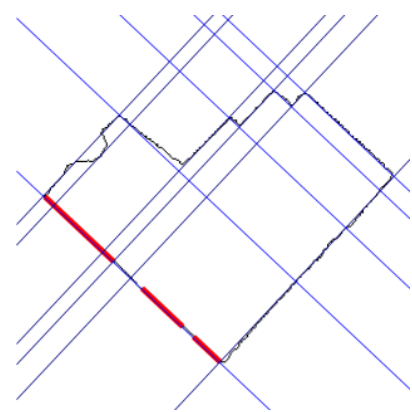

(c)

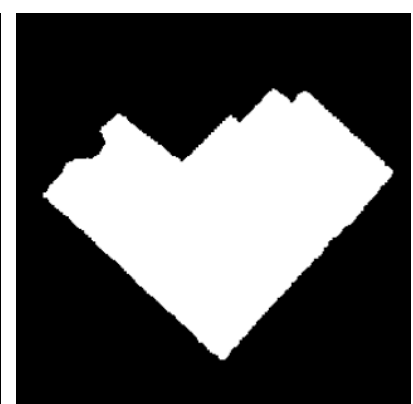

(b)

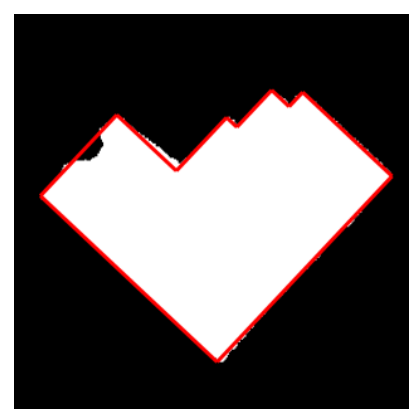

(d)
Figure 4 . The results of building outline extraction obtained by the raster approach for the selected building: (a) a rasterized point cloud of the building (extraction result is shown with a red outline); (b) a building mask after morphological closing;

(c) a building outline and perpendicular lines detected by Hough transform; (d) a vectorized outline.

\subsection{Quality Assessment}

Evaluation of the results has been done using the procedure proposed by Rutzinger et al. (2009). As a reference dataset, we used the building outlines that were obtained by manual interpretation of the initial UAV photogrammetric point cloud. The quality analysis was pixel-based; both reference outlines and extracted outlines were rasterised with a spatial resolution of $1 \mathrm{~cm}$. By comparing the extracted building outlines with the reference data, we retrieved the quantitative quality assessment about how much the extracted building outlines comply with the reference data. The quality assessment was determined with three quality measures: completeness, correctness, and quality:

- The completeness defines the percentage of the reference data that was detected in the extracted data.

- The correctness provides the percentage of extracted entities that correspond to the reference data.

- The quality is the measure that combines the completeness and correctness.

The described quality measures can be calculated by using the Equations (1), (2), and (3) (Rutzinger et al., 2009):

$$
\begin{aligned}
& \text { Completeness }=\frac{T P}{T P+F N} \\
& \text { Correctness }=\frac{T P}{T P+F P} \\
& \text { Quality }=\frac{T P}{T P+F P+F N}
\end{aligned}
$$

where: $\quad T P=$ true positive: a pixel classified as a building both in the reference data and in the evaluated data; $F N=$ false negative: a pixel classified as a background in the evaluated outlines but not in the reference;
$F P=$ false positive: a pixel classified as a building in the evaluated data, but not in the reference data.

\section{RESULTS AND DISCUSSION}

For both approaches, namely the direct approach and the raster approach, we conducted the building outline extraction from 5 point clouds, each having different point density as already presented. This yielded in 10 different datasets of building outlines; each resulting dataset contained 2D building polygons in a vector file format. Each building in the study area was represented with one $2 \mathrm{D}$ polygon, except in the case where two or more roofs of the building overlap with each other. The graphical results of the comparison of the extracted building outlines obtained by the direct approach with the reference data are shown in Figure 5. The graphical results of the comparison of the extracted building outlines obtained by the raster approach with the reference data are presented in Figure 6. Both Figures are showing outlines extracted from the point cloud with a density of $1 \mathrm{~cm}$.

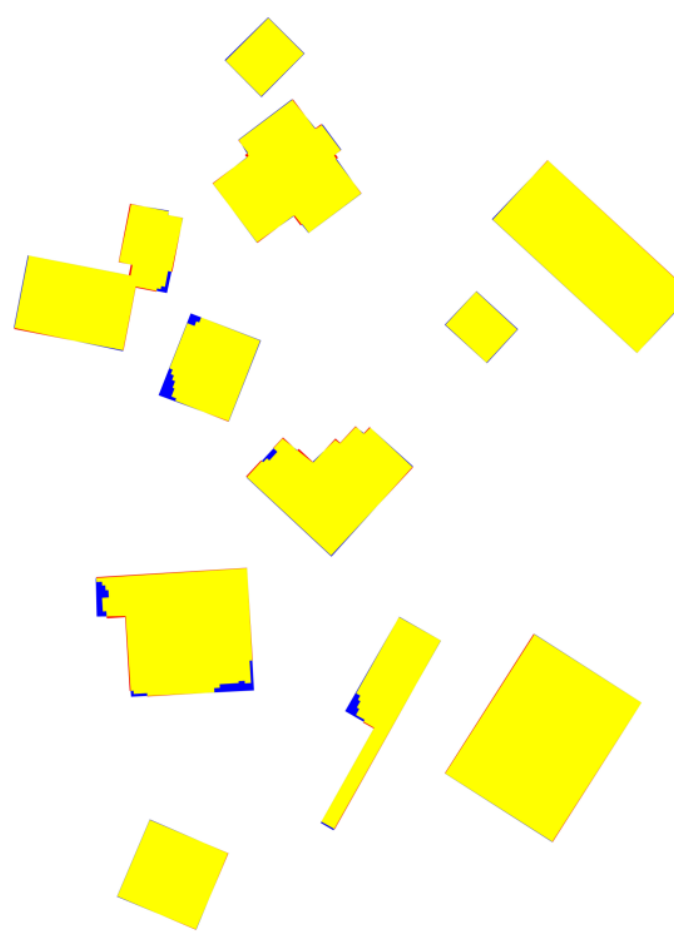

Figure 5. The extracted building outlines obtained by the direct approach from a point cloud with a density of $1 \mathrm{~cm}$ (meaning of colours: yellow - true positives; blue - false negatives; red false positives). 


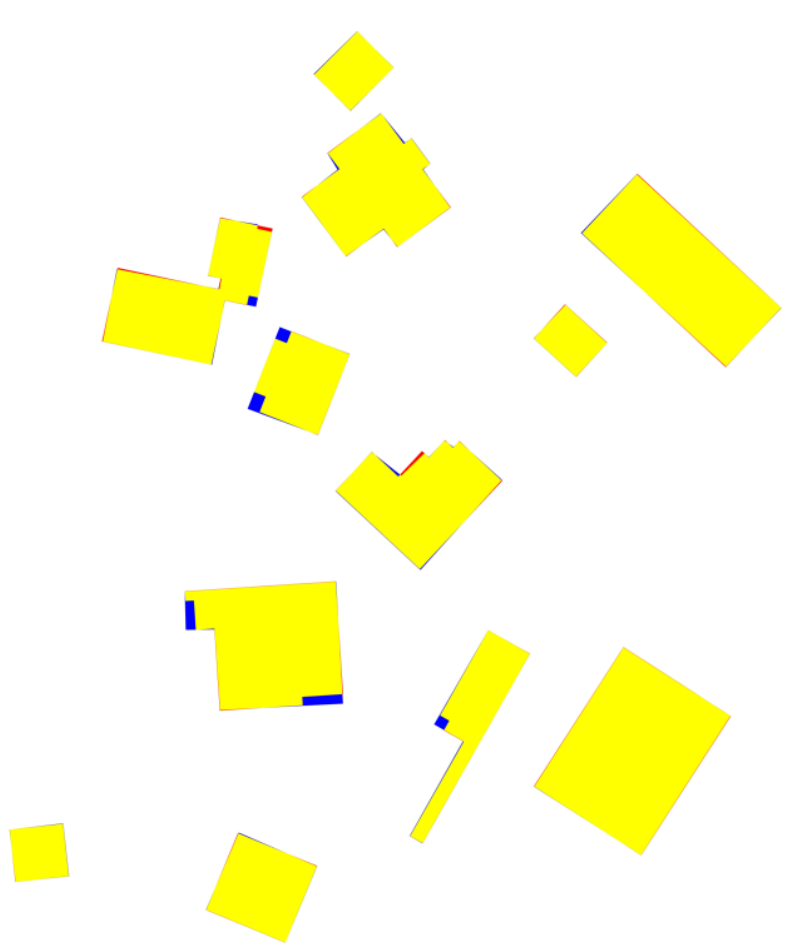

Figure 6 . The extracted building outlines obtained by the raster approach from a point cloud with a density of $1 \mathrm{~cm}$ (meaning of colours: yellow - true positives; blue - false negatives; red false positives).

If we compare the results from both building outline extraction approaches, we can see that the raster approach constructs more regular shapes of outlines than the direct approach. The sides of each outline are more consistent and follow the main orientations of the building. The outlines extracted by the direct approach are less regularised comparing to the raster approach, with more "zig-zag" lines that form the outline. Therefore, the raster approach is seen to be more robust to reconstruct outlines with lines that follow the main orientation of the building. We can further notice incomplete outlines of many buildings, especially in direct approach results, which is the consequence of missing points in the point cloud. However, independently of the approaches used for building outline extraction, the main disadvantage of using photogrammetric point cloud in the datadriven approach for 3D building modelling is the fact that some parts of the roofs might be missing in the point cloud due to shadows. Especially in areas, where high vegetation occludes part of the building, the points corresponding to the building are missing, because dense image matching is unable to provide the points below vegetation.

To evaluate the impact of point cloud density on the accuracy of the extracted building outlines, the quality of the outlines has been assessed. The quality assessment for both approaches and all point cloud densities is presented in Table 2 . The results show that, in general, the quality measures of all extracted outlines reached quite high values, almost all values are above $97 \%$. Here we have to keep in mind that the reference data used in the quality assessment was obtained with a manual vectorisation of the original input point cloud. This means that the quality should be regarded as a relative accuracy with respect to the input data. Relative accuracy enables us to evaluate only the impact of outline extraction approaches and point cloud density on the extracted building outlines. All other factors affecting the absolute accuracy of the outlines, such as the positional accuracy of input data, are not considered.

\begin{tabular}{|c|c|c|c|c|}
\hline \multirow{2}{*}{$\begin{array}{c}\text { Point } \\
\text { cloud } \\
\text { density }\end{array}$} & $\begin{array}{c}\text { Outline } \\
\text { extraction } \\
\text { approach }\end{array}$ & $\begin{array}{c}\text { Qompleteness } \\
{[\%]}\end{array}$ & $\begin{array}{c}\text { Correctness } \\
{[\%]}\end{array}$ & $\begin{array}{c}\text { Quality } \\
{[\%]}\end{array}$ \\
\hline \multirow{2}{*}{$\mathbf{1} \mathbf{~ c m}$} & DA & 97.94 & 99.30 & 97.26 \\
\cline { 3 - 5 } & RA & 98.44 & 99.38 & 97.85 \\
\hline \multirow{2}{*}{$\mathbf{2 . 5} \mathbf{~ c m}$} & DA & 97.95 & 99.25 & 97.23 \\
\cline { 2 - 5 } & RA & 98.58 & 99.30 & 97.90 \\
\hline \multirow{2}{*}{$\mathbf{5} \mathbf{~ c m}$} & DA & 97.98 & 99.29 & 97.29 \\
\cline { 2 - 5 } & RA & 98.57 & 99.23 & 97.82 \\
\hline \multirow{2}{*}{$\mathbf{1 0} \mathbf{~ c m}$} & DA & 97.72 & 99.52 & 97.26 \\
\cline { 2 - 5 } & RA & 98.09 & 99.05 & 97.18 \\
\hline \multirow{2}{*}{$\mathbf{2 0} \mathbf{~ c m}$} & DA & 97.31 & 99.47 & 96.80 \\
\cline { 2 - 5 } & RA & 97.59 & 99.14 & 96.77 \\
\hline
\end{tabular}

Table 2. The results of quality analysis of building outline extraction for all different point cloud densities $(1 \mathrm{~cm}, 2.5 \mathrm{~cm}, 5$ $\mathrm{cm}, 10 \mathrm{~cm}, 20 \mathrm{~cm}$ ) for both selected outline extraction approaches $(\mathrm{DA}=$ direct approach, $\mathrm{RA}=$ raster approach).

The graphical presentation of the quality measures is given in Figure 7 and Figure 8 . Figure 7 shows the values of completeness, correctness, and quality for extracted building outlines in case of using the direct approach for different point cloud densities.

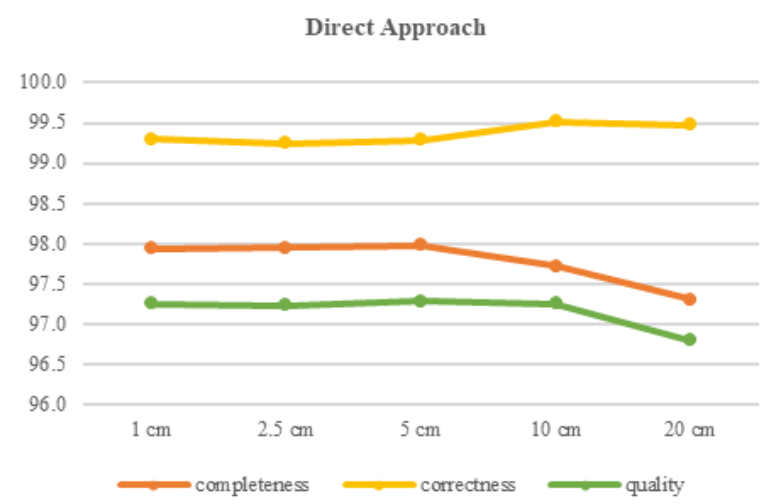

Figure 7 . The quality measures of the extracted building outlines using the direct approach for five different point cloud densities: $1 \mathrm{~cm}, 2.5 \mathrm{~cm}, 5 \mathrm{~cm}, 10 \mathrm{~cm}$, and $20 \mathrm{~cm}$.

In the direct approach for building outline extraction, for all point cloud densities, the correctness reached higher values than completeness. When analysing only completeness, we can see that it deteriorates when the point cloud density is decreasing. At the density of $1 \mathrm{~cm}$, the completeness was almost $98 \%$, and at the density of $20 \mathrm{~cm}$, it reached the value of $97.31 \%$. The difference in the completeness values between the highest and the lowest point cloud density is small, but the decreasing trend can be noticed. On the other side, the correctness achieved high values, around $99.5 \%$ regardless of the point cloud density. This means that the extracted outlines highly correspond to the reference data despite the different point cloud density. The values of quality as the third measure, which combines both the 
completeness and correctness, were also slightly affected by point cloud density. However, for densities from $1 \mathrm{~cm}$ to $10 \mathrm{~cm}$ the values are almost equal (around $97.25 \%$ ), decreasing to value $96.80 \%$ for the density of $20 \mathrm{~cm}$. In our opinion, the reason for this is mainly due to the settings of the used approach, where the values of parameters and tolerances in operations determine the quality of the obtained results.

The graphical presentation of the quality measures for building outline extraction with the raster approach for all point cloud density is given in Figure 8 .

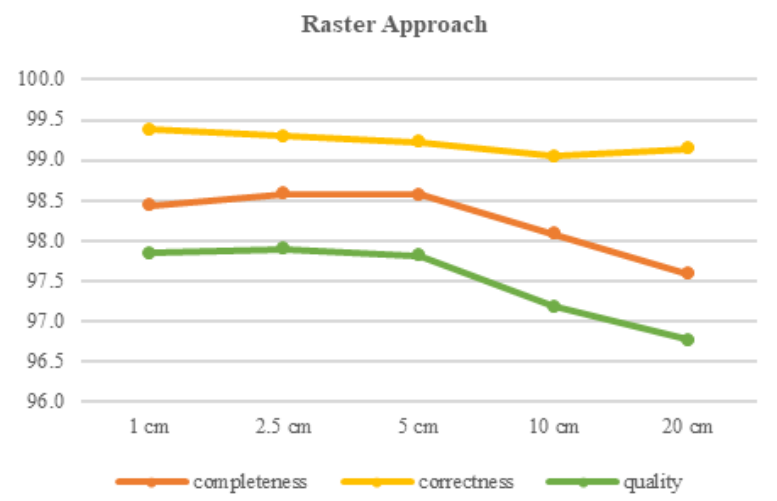

Figure 8 . The quality measures of the extracted building outlines using the raster approach for 5 different point cloud densities: $1 \mathrm{~cm}, 2.5 \mathrm{~cm}, 5 \mathrm{~cm}, 10 \mathrm{~cm}$, and $20 \mathrm{~cm}$.

We can see that in the raster approach, the values of correctness for all point cloud densities were higher than the values of completeness. For all point cloud densities, the correctness was a little bit below $99.5 \%$ and was not affected by the different point cloud density. On the contrary, the completeness values got lower when the point cloud density was sparser. At the density of $1 \mathrm{~cm}$, the completeness was $98.44 \%$, whereas at the density of $20 \mathrm{~cm}$ it decreased to $97.59 \%$. Similarly, as in the direct approach, the value of quality was also affected by the point cloud density, reaching the values $97.85 \%$ at the density of $1 \mathrm{~cm}$ and $96.77 \%$ at the density of $20 \mathrm{~cm}$. The quality obtained lower values in case of sparser point clouds as a consequence of lower completeness. Similarly, as in the direct approach, the effect of lower point cloud density on the quality of extracted building outlines can be seen when using the raster approach. In case of a dense point cloud, having the density $5 \mathrm{~cm}$ or better, the outlines are extracted with similar quality, so we can say that the density is not crucial factor that affects the quality of outlines in those cases. When the density is further reduced, either to $10 \mathrm{~cm}$ or $20 \mathrm{~cm}$, the quality and completeness deteriorate, so the point cloud density affects the results.

\section{CONCLUSIONS}

In our research, we investigated the impact of point cloud density on the quality of the extracted building outlines from the UAV photogrammetric point cloud data. We used two approaches for outline extraction, the direct and the raster approach, where each of the approaches generates the outlines with its operations. The quality assessment was determined with three quality measures: completeness, correctness, and quality. Although the processing steps in each of the selected approaches are different, we got similar results in both cases. However, the raster approach, in general, gives slightly better numerical results than the direct approach (Table 2). Also, in the graphical presentation of the results (Figures 5 and 6), we can see that the raster approach constructs more regular shapes of outlines than the direct approach. Further on, we can only partly confirm that the density of point cloud impacts the extraction of building outline. For quality measure, there is no significant difference for densities from $1 \mathrm{~cm}$ to $10 \mathrm{~cm}$ using the direct approach, and for densities from $1 \mathrm{~cm}$ to $5 \mathrm{~cm}$ using a raster approach. Similar conclusions can be observed in the results for completeness measure. This is in line with the general properties of the point cloud and the possibility to extract entities from it. In more dense point cloud, the features' boundary, such as roof edge, is defined more precisely, which means that it can be extracted with greater completeness.

On the contrary, for the correctness measure of the building outlines in our case study, we can not confirm that point cloud density (from $1 \mathrm{~cm}$ to $20 \mathrm{~cm}$ ) is significantly affecting the results. This means that the features which were extracted are in line with entities in the scene regardless of the point cloud density we used.

In our building outline extraction workflow, the main limitation of using the photogrammetric point cloud was the presence of vegetation that occlude the buildings, which causes the incomplete point cloud and consequently incomplete outline.

The conducted analysis of point cloud density impact on the building outline extraction has given us some insight into how the quality of building outlines changes with respect to the different point cloud densities. Since our study area includes only a few and not much-occluded buildings by vegetation, the quality measures obtained high values. For more detailed analysis, the proposed methodology should be used on a larger dataset. Further research could investigate the impact of the different parameters' values, which define the two selected approaches for building outline extraction.

\section{ACKNOWLEDGEMENTS}

The authors acknowledge the financial support of the Slovenian Research Agency (research core funding No. P2-0406 Earth observation and geoinformatics).

\section{REFERENCES}

Albers, B., Kada, M., Wichmann, A., 2016. Automatic extraction and regularization of building outlines from airborne Lidar point clouds. International Archives of the Photogrammetry, Remote Sensing and Spatial Information Sciences, XLI-B3, 555-560. doi.org/10.5194/isprsarchivesXLI-B3-555-2016.

Awrangjeb, M., Lu, G., 2014. Automatic building footprint extraction and regularisation from LIDAR point cloud data. International Conference on Digital Image Computing: Techniques and Applications (DICTA) 2014, 1-8. doi.org/10.1109/DICTA.2014.7008096.

Biljecki, F., Stoter, J., Ledoux, H., Zlatanova, S., Çöltekin, A., 2015. Applications of 3D city models: state of the art review. ISPRS International Journal of Geo-Information, 4(4), 2842 2889. doi.org/10.3390/ijgi4042842. 
Dai, Y., Gong, J., Li, Y., Feng, Q., 2017. Building segmentation and outline extraction from uav image-derived point clouds by a line growing algorithm. International Journal of Digital Earth 10(11), 1077-1097. doi.org/10.1080/17538947.2016.1269841.

Dorninger, P., Pfeifer, N., 2008. A comprehensive automated 3D approach for building extraction, reconstruction, and regularization from airborne laser scanning point clouds. Sensors, 8(11), 7323-7343. doi.org/10.3390/s8117323.

dos Santos, R. C., Galo, M., Carrilho, A. C., 2019. Extraction of Building Roof Boundaries from LiDAR Data Using an Adaptive Alpha-Shape Algorithm. IEEE Geoscience and Remote Sensing Letters, 16(8), 1289-1293. doi.org/10.1109/LGRS.2019.2894098.

Gilani, S. A. N., Awrangjeb, M., Lu, G., 2016. An automatic building extraction and regularisation technique using LiDAR point cloud data and orthoimage. Remote Sensing, 8(3), 258. doi.org/10.3390/rs8030258.

Gonzalez, R. C., Woods, R. E., Eddins, S. L., 2004. Digital image processing using Matlab. Pearson Prentice Hall, New Jersey.

Grigillo, D., Kanjir, U., 2012. Urban object extraction from digital surface model and digital aerial images. ISPRS Annals of the Photogrammetry, Remote Sensing and Spatial Information Sciences, I-3, 215-220. doi.org/10.5194/isprsannals-I-3-2152012.

Haala, N., Kada, M., 2010. An update on automatic 3D building reconstruction. ISPRS Journal of Photogrammetry and Remote Sensing, 65(6), 570-580. doi.org/10.1016/j.isprsjprs.2010.09.006.

Habib, A. F., Zhal, R., Kim, C., 2010. Generation of complex polyhedral building models by integrating stereo-aerial imagery and lidar data. Photogrammetric Engineering \& Remote Sensing, 76(5), 609-623. doi.org/10.14358/PERS.76.5.609.

Jarzabek-Rychard, M., 2012. Reconstruction of building outlines in dense urban areas based on LIDAR data and address points. International Archives of the Photogrammetry, Remote Sensing and Spatial Information Sciences, XXXIX-B3, 121126. doi.org/10.5194/isprsarchives-xxxix-b3-121-2012.

Jarzabek-Rychard, M., Maas, H. G., 2017. Geometric refinement of ALS-data derived building models using monoscopic aerial images. Remote Sensing, 9(3), 282. doi.org/10.3390/rs9030282.

Kaartinen, H., Hyyppä, J., Gülch, E., Hyyppä, H., Matikainen, L., Vosselman, G., Hofmann, A. D., Mäder, U., Persson, Å., Söderman, U., Elmqvist, M., Ruiz, A., Dragoja, M., Flamanc, D., Maillet, G., Kersten, T., Carl, J., Hau, R., Wild, E., Frederiksen, L., Holmgaard, J., Vester, K., 2005. EuroSDR building extraction comparison. In: ISPRS Hannover Workshop 2005: High-Resolution Earth Imaging for Geospatial Information. Hannover, Germany, 17-20 May 2005.

Li, M., Nan, L., Smith, N., Wonka, P., 2016. Reconstructing building mass models from UAV images. Computers and Graphics, 54, 84-93. doi.org/10.1016/j.cag.2015.07.004.

Malihi, S., Zoej, M. J. V., Hahn, M., 2018. Large-scale accurate reconstruction of buildings employing point clouds generated from UAV imagery. Remote Sensing, 10(7), 1148. doi.org/10.3390/rs10071148.

Pfeifer, N., Rutzinger, M., Rottensteiner, F., Muecke, W., Hollaus, M., 2007. Extraction of building footprints from airborne laser scanning: Comparison and validation techniques. In: 2007 Urban Remote Sensing Joint Event. Paris, France, 1113 April 2007. doi.org/10.1109/URS.2007.371854.

Rottensteiner, F., Sohn, G., Gerke, M., Wegner, J. D., Breitkopf, U., Jung, J., 2014. Results of the ISPRS benchmark on urban object detection and 3D building reconstruction. ISPRS Journal of Photogrammetry and Remote Sensing, 93, 256-271. doi.org/10.1016/j.isprsjprs.2013.10.004.

Rutzinger, M., Rottensteiner, F., Pfeifer, N., 2009. A comparison of evaluation techniques for building extraction from airborne laser scanning. IEEE Journal of Selected Topics in Applied Earth Observations and Remote Sensing, 2(1), 1120. doi.org/10.1109/JSTARS.2009.2012488.

Sampath, A., Shan, J., 2007. Building boundary tracing and regularization from airborne lidar point clouds. Photogrammetric Engineering and Remote Sensing, 73(7), 805-812. doi.org/10.14358/PERS.73.7.805.

Tomljenovic, I., Höfle, B., Tiede, D., Blaschke, T., 2015. Building extraction from Airborne Laser Scanning data: An analysis of the state of the art. Remote Sensing, 7(4), 38263862. doi.org/10.3390/rs70403826.

Widyaningrum, E., Gorte, B., Lindenbergh, R., 2019. Automatic building outline extraction from ALS point clouds by ordered points aided hough transform. Remote Sensing, 11(14), 1727. doi.org/10.3390/rs11141727.

Zhang, K., Yan, J., Chen, S. C., 2006. Automatic construction of building footprints from airborne LIDAR data. IEEE Transactions on Geoscience and Remote Sensing, 44(9), 25232533. doi.org/10.1109/TGRS.2006.874137. 